\title{
A Plant-Based Meal Reduces Postprandial Oxidative and Dicarbonyl Stress in Men with Diabetes or Obesity more than an Energy- and Macronutrient- Matched Conventional Meal in a Randomized Crossover Study
}

Hana Malinska

IKEM

Marta Klementova

IKEM

Michaela Kudlackova

IKEM

Jiri Veleba

IKEM

Eva Hoskova

IKEM

Olena Oliyarnyk

IKEM

Irena Markova

IKEM

Lenka Thieme

IKEM

Martin Hill

Institute of Endocrinology

Terezie Pelikanova

IKEM

Hana Kahleova ( $\nabla$ hana.kahleova@gmail.com )

Physicians Committee for Responsible Medicine https://orcid.org/0000-0003-0491-3993

Research Article

Keywords: Postprandial state, type 2 diabetes, plant-based diet, inflammation, methylglyoxal, oxidative stress, appetite hormones 
Posted Date: May 24th, 2021

DOI: https://doi.org/10.21203/rs.3.rs-491370/v1

License: (c) (i) This work is licensed under a Creative Commons Attribution 4.0 International License. Read Full License 


\section{Abstract}

Background: Increased oxidative/dicarbonyl stress and chronic inflammation are considered key pathological mediators in the progression of complications in type 2 diabetes (T2D) and obesity. Lifestyle and diet composition have a major impact. In this study, we tested the effects of a vegan $(\mathrm{V})$ and a conventional meat containg (M) meal, matched for energy and macronutrients, on postprandial oxidative and dicarbonyl stress, inflammatory markers and appetite hormones.

Methods: A randomised crossover design was used to evaluate T2D, obese and control participants ( $n=20$ in each group), with serum concentrations of analytes determined at 0,120 and 180 minutes. Repeated-measures ANOVA was used for statistical analysis.

Results: Postprandial glucose, triglycerides and free fatty acid responses were similar after both meals. After the V-meal consumption, we observed decreased postprandial concentrations of oxidised glutathione $(p \otimes 0.001)$ and increased glutathione peroxidase activity $(p=0.045)$ compared with the M-meal in T2D subjects. In obese participants, V-meal consumption increased postprandial concentrations of reduced glutathione $(p=0.041)$ and decreased methylglyoxal concentrations $(p=0.023)$. There were no differences in postprandial secretion of TNFa, MCP-1 or ghrelin, but we did observe higher postprandial secretion of leptin after the V-meal in T2D subjects $(p=0.002)$ compared with the M-meal.

Conclusions: The results show that a plant-based meal is efficient in ameliorating the postprandial oxidative and dicarbonyl stress compared to a conventional energy- and macronutrient-matched meal, indicating the therapeutic potential of plant-based nutrition in improving the progression of complications in T2D and obese patients.

Registered under ClinicalTrials.gov Identifier No. NCT02474147.

\section{Introduction}

Type 2 diabetes (T2D) and obesity are associated with postprandial dysmetabolism, a state that significantly contributes to the development of associated complications [1]. It has been recently suggested that non-fasting glucose and, particularly, postprandial concentrations of blood glucose and lipids are reliable predictors of cardiovascular disease [2,3]. A prolonged postprandial period may induce pro-oxidant conditions and pro-inflammatory activity, both of which are implicated in micro- and macrovascular damage [1].

Abnormal postprandial elevation of plasma glucose and lipids in T2D is associated with oxidative stress, increased inflammation, hypercoagulation and impaired secretion of gastrointestinal hormones $[4,5]$. Our previous findings from a study involving T2D patients showed that postprandial oxidative stress occurred independently of alterations in gastrointestinal peptides [6]. It has been recently suggested that postprandial formation of dicarbonyl compounds, toxic reactive metabolites from glucose and lipids, may be involved in the development of vascular complications of diabetes [7]. Dicarbonyls interact with 
proteins to form advanced glycation end products, which can damage the endothelium and impair vasorelaxation [8].

The management of postprandial dysmetabolism represents an important strategy in the treatment of T2D and its complications. One important element of this approach is diet composition. The preventative and therapeutic effects of vegetarian diets on diabetes have been examined over recent decades and have been shown at least as beneficial as pharmacotherapy in diabetes management [9]. Vegetarian diets are inversely associated with the risk of developing diabetes and are associated with two-fold lower the prevalence of T2D [10]. In a previous clinical interventional study, a vegetarian diet led to greater weight loss and improved glycaemic control, insulin resistance and oxidative stress markers compared to a conventional hypocaloric diet [11]. Furthermore, our previous randomised crossover study reported that a plant-based meal increased postprandial secretion of gastrointestinal hormones more effectively than a proceseed meat meal in obese and T2D men [12]. Another study found improvements in postprandial incretin and insulin secretion after a plant-based meal in T2D patients, highlighting the therapeutic potential of this type of diet in improving b-cell function [13]. In this secondary analysis of previously published data [14], we have tested postprandial response to a plant-based meal compared with an energy- and macronutrient-matched conventional meal on oxidative and dicarbonyl stress, inflammation and appetite hormones in T2D and obese patients. Our hypothesis was that a plant-based meal would stimulate postprandial appetite hormones secretion and produce lower postprandial response of oxidative and dicarbonyl stress compared to the conventional meal. The results of the study may help build the evidence base for dietary guidelines for T2D and obese patients.

\section{Materials And Methods}

\section{Study subjects and design}

The methods have beedn described in detail previously [14]. Breinfly: This randomised crossover study group included 20 men with T2D, 20 obese men and 20 healthy men. All individuals consumed two energy- and macronutrient-matched test meals in random order. The general metabolic characteristics of each group are given in Table 1. Written informed consent was obtained from all participants prior to enrolment in the study. The study was approved by the ethics committees of Thomayer Hospital and the Institute for Clinical and Experimental Medicine, Prague, Czech Republic (protocol identification number G14-08-42). The study was prospectively registed at ClinicalTrials.gov (Identifier: NCT02474147).

All participants were male and of Czech nationality. Men with T2D and at least three hallmarks of metabolic syndrome (30-65 years of age; BMI, $25-45 \mathrm{~kg} / \mathrm{m}^{2} ; \mathrm{HbA} 1 \mathrm{c}, 42-105 \mathrm{mmol} / \mathrm{mol}$ ) were treated by lifestyle alone or with oral hypoglycaemic agents (metformin and/or sulfonylureas) for at least one year. The obese men were BMI- and age-matched to men with T2D; the healthy men comprised age-matched controls with normal BMI (between 19 and $25 \mathrm{~kg} / \mathrm{m}^{2}$ ) and normal glucose tolerance. Exclusion criteria were thyroid, liver or kidney disease, drug or alcohol abuse, unstable drug therapy, or significant weight loss of more than $5 \%$ body weight in the preceding three months. 


\section{Randomization and interventions}

The participants were randomly assigned in a 1:1 ratio a vegan meal or an energy- and marconutrientmatched conventional meat containing meal based on a computer-generated randomisation protocol. The randomization protocol was designed so as not to be accessed beforehand, with the interventions unmasked. Outcome assessors were blinded to the interventions.

All participants fasted for 10 to 12 hours overnight. Diabetic patients avoided diabetes medication the evening before, and on the morning of, the assessment. The meal consisted of either a conventional meat burger (M-meal) or a vegan burger (V-meal). The composition of the test meals is given in Table 2. Tap water was allowed ad libitum. Unaware of the sequence of interventions, participants arrived at the laboratory in the morning to be assigned one of the randomized test meals. The participants were checking in for their meal assessments between 7-8:30 am. They usually finished their meal in 15-20 mins. The whole meal test took 3 hours from finishing their meal. After a washout period of one week, participants returned to complete the opposite test meal. The participants were instructed not to change their usual dietary habits or physical activity during the study. All participants found both meals acceptable and nobody complained about any particular meal.

\section{Analytical methods}

Anthropometric measurements and blood pressure: Height and weight were measured using a stadiometer with a calibrated scale accurate to $0.1 \mathrm{~kg}$. Ensuring participants had been in a seated position be forehand, blood pressure was measured using the M6 Comfort digital monitor on three occasions at 2-minute intervals. A mean value was calculated from the last two measurements. Blood samples were drawn in the fasting state and then 30,60, 120 and 180 minutes after the standard meal. After that, the samples were centrifuged and aliquots of plasma/serum were stored at $-80^{\circ} \mathrm{C}$ for analysis. Plasma glucose was analysed using the Beckman Analyzer glucose-oxidase method (Beckman Instruments, Inc., Fullerton, CA, USA) and glycated haemoglobin using the VARIANT II Hemoglobin Testing System (Bio-Rad Laboratories $\mathrm{GmbH}$, Munich, Germany). Plasma lipids were measured using enzymatic methods (Roche, Basel, Switzerland).

Inflammatory markers and appetite hormones: Concentrations of TNFa, MCP-1, leptin and ghrelin were determined by multiplex immunoanalysis based on XMAP technology using the MILLIPLEX MAP Human Metabolic Hormone Magnetic Bead Panel (HMHEMAG-34K) (Millipore, Billerica, MA, USA) and the Luminex 100 IS instrument (Luminex Corporation, Austin, USA).

Oxidative stress markers: The whole blood level of reduced and oxidised forms of glutathione were determined using the Glutathione in Whole Blood - HPLC diagnostic kit (Chromsystems, Munich, Germany). The activity of glutathione peroxidase (GPx) was analysed using a glutathione peroxidase assay kit (Cayman Chemical, MI, USA). The serum level of ascorbic acid was measured using a spectrophotometric method as previously described [6]. 
Dicarbonyl stress markers: The concentration of methylglyoxal was determined after derivatisation with 1,2-diaminobenzene using HPLC and fluorescence detection according to Fleming and Bierhaus as previously described [15]. A registered dietitian analyzed both meals, using a countr-speficif food database and software [16].

\section{Statistical analysis}

Sample size was estimated based on a power analysis with an alpha of 0.05 and a power of 0.80 to detect between intervention differences in thalamus perfusion (primary outcome) and serum concentrations of GLP-1 and GSSH (secondary outcomes), using the PASS 16.0 Power analysis and sample size software, 2018 (NCSS, Kaysville, UT, USA). Based on our preliminary data, after data transformation to achieve normal distribution, to have $80 \%$ power to detect a difference between the two meals would require 10 subjects in each group for the primary outcome and 14 subjects in each group were required for the secondary outcomes. Assuming an attrition of $25 \%$, the expected sample size is 18 in each group.

Intention to treat analysis was performed, using repeated-measures ANOVA. Group, subject and time factors were all included in the model as follows: inter-individual (T2D vs. obese vs. controls); intraindividual (time taken to complete the meal test); and interaction between factors (divergence degree between the time profiles of each group).

To eliminate skewed data distribution and heteroscedasticity, the original data was transformed to a Gaussian distribution to attain symmetric distribution in both predictors and dependent variables and, at the same time, to stabilize the variance (attaining homoscedasticity), by a power transformation using the statistical software Statgraphics Centurion, version XV from Statpoint Inc. (Herndon, Virginia, USA), as descibed in detail previously [17].

Spearman's correlations were calculated based on the relationship between postprandial changes in oxidative/dicarbonyl stress markers and alterations in inflammatory markers and appetite hormones. The fasting period in addition to any changes (120-0, and 180-120 min after ingestion of a standard breakfast) were calculated as follows: for each separate period, and then for all 5 values combined. Analysis was carried out using PASS 2005 statistical software (Number Cruncher Statistical Systems, USA); the statistician was blinded to the analysis. Data are presented as the mean with a $95 \%$ confidence interval (Cl).

\section{Results}

The flow-chart showing the recruitment and follow-up of the participants is in Figure S1. The general characteristics for the individual groups of participants are shown in Table 1, with the composition of meals shown in Table 2. 
As shown in Figure 1, there were no differences in plasma concentrations of glucose, triglycerides or free fatty acids between the M-meal and V-meal across all groups during the postprandial state. However, in T2D and obese subjects, we observed increased basal fasting levels in conjunction with higher changes and dynamics in the postprandial state for these parameters irrespective of test meal. Plasma concentrations of triglycerides were inversely related to concentrations of free fatty acids.

\section{Oxidative and dicarbonyl stress parameters}

In general, after the plant-based V-meal, we observed improvements in oxidative and dicarbonyl stress parameters in the postprandial state, particularly in T2D patients (Figure 2). We observed significantly decreased levels of the oxidised form of glutathione (GSSG) and increased GPx activity in T2D patients after the V-meal compared to the M-meal. After the V-meal compared to M-meal in obese subjects, we observed an increase in postrandial levels of glutathione and reduced postprandial concentrations of methylglyoxal. In T2D patients, there were no significant differences in methylglyoxal levels between meal types. In all groups of participants, the changes in postprandial levels of methylglyoxal are not excesively different between meal types. However, postprandial methylglyoxal levels were markedly higher in terms of quantity as well as dynamics in men with T2D compared with obese and control subjects. In T2D patients V-meal compared to M-meal significantly increased the activity of glutathionedependent antioxidant enzyme GPx that can associated with postmeal glutathione status after V-meal. In controls, postprandial levels of ascorbic acid were more elevated after the V-meal compared to the Mmeal. The post-meal levels of ascorbic acid in T2D and obese participants were lower than in healthy controls irrespective of test meal.

\section{Inflammatory parameters and appetite hormones}

Postprandial secretion of pro-inflammatory TNFa and MCP-1 in T2D and obese participants was not affected by the test meal consumed. However, compared to controls, in T2D subjects we observed markedly increased post-meal levels of both pro-inflammatory markers, while in obese subjects we observed increased MCP-1 concentrations only in the postprandial state. In control subjects, V-meal compared to M-meal significantly increased postprandial levels of TNFa, however, compared to T2Ds levels of TNFa in controls are markedly decreased.

The concentrations and post-meal amplitudes of the appetite hormones leptin and ghrelin differed significantly between T2D, obese and healthy subjects. Plasma concentrations of leptin were markedly elevated in T2D and obese subjects, while plasma concentrations of ghrelin were reduced compared to healthy controls. The postprandial dynamics of ghrelin in controls were far more pronounced than in T2D and obese patients. The concentrations of leptin in the postprandial state were siginificantly higher after the V-meal in T2Ds as well as in control subjects. These results are presented in Figure 3.

\section{Discussion}


Diet and its composition play an important role in influencing the postprandial state. In conjunction with other lifestyle changes, nutrition is recommended for preventing the development of complications associated with onset of diabetes and obesity. Based on our previous results, a plant-based meal stimulates postprandial insulin secretion more than a conventional meat meal in men with T2D [18], as is the case for postprandial secretion of gastrointestinal hormones, particularly incretins [13]. These results suggest that a plant-based diet may improve b-cell function [13].

\section{Oxidative and dicarbonyl stress}

The increase in postprandial oxidative and dicarbonyl stress was less pronounced after the V-meal compared with the M-meal. The most prominent differences between both meals occurred in respect of postprandial responses of glutathione and methylglyoxal. The V-meal reduced postprandial amplitudes of oxidative and dicarbonyl stress more than the M-meal. This suggests that a vegan nutrition may offer better protection against the generation of toxic metabolites involved in the development of complications caused by postprandial hyperglycaemia and hyperlipidaemia. One such toxic metabolite is methylglyoxal. This highly reactive dicarbonyl compound has emerged as a biomarker of diabetes and is closely associated with protein glycation and insulin resistance [19]. The generation of toxic metabolites during hyperglycaemia and hyperlipidaemia is implicated in the early development of diabetic complications whereby metabolites persist even after glucose and lipid normalization [20]. In our study, we have measured postprandial changes of methylglyoxal in T2D and obese individuals, although the differences between the meals were relatively small. However, in T2D patients, postprandial levels of methylglyoxal were markedly elevated compared to obese and control subjects, thus increasing cardiovascular risk. Decreased levels of reduced glutathione in T2D patients can induce both oxidative as well dicarbonyl stress, while glutathione is involved in methylglyoxal degradation as a co-factor of glyoxalase-1 $[7,8]$.

Bioactive compounds such as polyphenols and other antioxidants might be an important element in the beneficial effects of a plant-based diet on oxidative stress. Dietary polyphenols, a large and heterogeneous group of bioactive compounds, exhibit protective antioxidant properties and also activate transcriptional factor Nrf2. The Nrf2/ARE signalling pathway is an important defence system against exogenous and endogenous oxidative stress injury [21]. In addition to their beneficial dietary effects, polyphenols are understood to possess immunomodulatory, anti-inflammatory [22], and even antidiabetic properties [23]. Research in this emerging area is ongoing. To the best of our knowledge, a study comparing the post-meal response of methylglyoxal to oxidative stress parameters in T2D patients has not yet to be published.

V-meal consumed in our study increased postprandial response in GPx activity in T2D patients, but there were no differences between the groups of participants. We assumed this effect has only minor impact on the pathology of diabetes and its complications. On the other hand, differences in postprandial response of V-meal compared to $\mathrm{M}$-meal in glutathione can more affect postprandial oxidative stress and can also affect GPx activity. Although we observed decreased levels of postprandial ascorbic acid in T2D 
and obese individuals, the meal type consumed did not affect the postprandial response in either group. It has been shown that glucose and ascorbic acid compete for entry into cells; therefore, any postprandial decrease in ascorbic acid would be more associated with hyperglyceamia than hyperlipidaemia, where glucose inhibits the input of ascorbic acid into cells [24]. It is also interesting that postprandial levels of ascorbic acid and glutathione have opposite relationship to each other in dynamics independently to type of meal.

\section{Inflammation and appetite hormones}

The postprandial state is a condition characterised by low-grade inflammation, whereby cells respond to acute elevations of carbohydrates, triglycerides and fatty acids [25]. Previous studies have shown that post-meal hyperlipidaemia and hyperglycaemia increase TNFa levels in healthy and T2D individuals [26] as well as in IGT subjects [27]. In our study, T2D patients exhibited increased postprandial levels of TNFa and MCP-1, with only MCP-1 levels increasing in obese individuals. Therefore, postprandial MCP-1 levels may not only be a more sensitive marker but also a better early predictor of low-grade inflammation than TNFa, particularly in non-diabetic patients. However, no differences were observed in MCP-1 following the ingestion of different meals across groups. The slightly increased postprandial secretion of TNFa in the control group following the V-meal may have been due to the proportion of soy protein content. Soybean meal protein can induce an inflammatory response by increasing the secretion of pro-inflammatory cytokines $[28,29]$. Although increased TNFa following the V-meal was only observed in the control group, general TNFa levels and postprandial amplitudes in controls were all lower than those in T2D and obese subjects. On the other hand, soy protein contains high amounts of arginine and glycine, amino acids associated with decreased cholesterol levels [30,31].

The obese and T2D patients in our study exhibited diminished post-meal suppresion of ghrelin secretion and markedly increased postprandial levels of leptin. This finding corresponds with previous results reporting postprandial changes in ghrelin to be negatively associated with plasma triglycerides [6]. Plantbased meal better stimulated postprandial secretion of leptin than conventional meat meal. While the action of leptin is essential for energy metabolism, it is also involved in the lipid mobilisation of different fat depots and is understood to protect tissues during lipotoxicity [32]. Lipid oxidation can increase via leptin signalling and has been reported to reduce excess fatty acids [33]. In our study, the increased postprandial response of leptin after the V-meal may have led to such effects. As previously described [18], a plant-based diet increases satiety in T2D patients, while the postprandial response of the appetite hormones leptin and ghrelin can also contribute to feeling full.

\section{Strengths, limitations and interpretation}

In comparison with the standard oral glucose tolerance test, the two different sandwich meal tests given to the participants in our study contained all of the main nutrient groups, thus increasing physiological stimulation during the post-meal response. This enabled us to identify the mechanism that reflects more than just glucose metabolism. In addition, both meals were served in amounts usually ingested during a typical meal, rendering our results highly applicable. Representing one of the limitations of our study, we 
did not account for the habitual diets and dietary patterns of the participants when investigating their acute post-meal responses. Also, a longer-term study would be more accurate in determining the merits of a plant-based diet in slowing down and preventing the progression of complications in T2D and obese patients. Nevertheless, we were able to reveal differences in postprandial metabolic mechanisms by comparing single-meal responses, which may assist futher research into retarding or preventing the development of associated complications. Also, dietary habits and nutritional status of participants may influence basal levels of some parameters observed during meal tests.

Oxidative and dicarbonyl stress is a key mechanism in the development of vascular complications during the post-meal phase and should be considered a separate therapeutic target for pharmacological as well as dietary/nutrition interventions aimed at preventing such complications. A plant-based nutrition may be therefore recommended for T2D and obese patients.

In conclusion, our results indicate that the plant-based meal ameliorated the exacerbation of post-meal oxidative and dicarbonyl stress in T2D and obese men compared to the conventional meat meal, and thus can provide better protection against the development of complications associated with diabetes and obesity. However, further studies are needed to verify these effects, in particular after long-term adherence to a plant-based diet.

\section{Abbreviations}

GPx - glutathione peroxidase, GSSG - oxidised form of glutathione, MCP-1 - monocyte chemoattractant protein 1, NEFA - non-esterified fatty acids, Nrf2 - nuclear factor-erythroid 2-related factor-2, TNFa - tumour necrosis factor a

\section{Declarations}

Ethics approval and consent to participate: Written informed consent was obtained from all participants prior to enrolment in the study. The study was approved by the ethics committees of Thomayer Hospital and the Institute for Clinical and Experimental Medicine, Prague, Czech Republic (protocol identification number G14-08-42). All participants gave informed, written consent.

Consent for publication: All authors had full access to the data and approved the final version of the manuscript for publication.

Availability of data and materials: The deidentified data will be available upon request at hkahleova@pcrm.org

Competing Interests: No conflict of interest related to this manuscript has been reported.

Funding: The study was supported by the Ministry of Health of the Czech Republic through grant project AZV15-27338A and the Conceptual Development of Research Organisations program (Institute for 
Clinical and Experimental Medicine - IKEM, IN 00023001).

Authors' contributions: TP and HK designed the study and wrote the grant application and study protocol. MK (Marta Klementova), MK (Michaela Kudlackova), JV, EH and LT recruited the patients, randomised the meal test order and collected the data. $\mathrm{HM}, \mathrm{OO}$ and IM acquired and analysed the data. MH was responsible for statistical analysis and interpretation of data. HM and HK drafted the manuscript. All authors had full access to data, critically revised the article and approved the manuscript for publication.

Acknowledgements: We thank the study participants for their invaluable contribution.

\section{References}

1. Sottero B, Gargiulo S, Russo I, Barale C, Poli G, Cavalot F. Postprandial Dysmetabolism and Oxidative Stress in Type 2 Diabetes: Pathogenetic Mechanisms and Therapeutic Strategies. Med Res Rev. 2015;35:968-1031.

2. Node $\mathrm{K}$, Inoue T. Postprandial hyperglycemia as an etiological factor in vascular failure. Cardiovasc Diabetol. 2009;8:23.

3. Pang J, Chan DC, Barrett PH, Watts GF. Postprandial dyslipidaemia and diabetes: mechanistic and therapeutic aspects. Curr Opin Lipidol. 2012;23:303-9.

4. Ceriello A, Esposito K, Testa R, Bonfigli AR, Marra M, Giugliano D. The possible protective role of glucagon-like peptide 1 on endothelium during the meal and evidence for an "endothelial resistance" to glucagon-like peptide 1 in diabetes. Diabetes Care. 2011;34:697-702.

5. Nauck MA, Meier JJ. Incretin hormones: Their role in health and disease. Diabetes Obes Metab. 2018;20(Suppl 1):5-21.

6. Malinska H, Kahleova H, Topolcan O, Vrzalova J, Oliyarnyk O, Kazdova L, Belinova L, Hill M, Pelikanova T. Postprandial oxidative stress and gastrointestinal hormones: is there a link? PLoS One. 2014;9:e103565.

7. Hanssen NMJ, Scheijen J, Houben A, van de Waarenburg M, Berendschot T, Webers CAB, Reesink KD, van Greevenbroek MMJ, van der Kallen C, Schaper NC, et al. Fasting and post-oral-glucose-load levels of methylglyoxal are associated with microvascular, but not macrovascular, disease in individuals with and without (pre)diabetes: The Maastricht Study. Diabetes. Metab. 2020.

8. Schalkwijk CG. Vascular AGE-ing by methylglyoxal: the past, the present and the future. Diabetologia. 2015;58:1715-9.

9. Olfert MD, Wattick RA. Vegetarian Diets and the Risk of Diabetes. Curr Diab Rep. 2018;18:101.

10. Tonstad S, Butler T, Yan R, Fraser GE. Type of vegetarian diet, body weight, and prevalence of type 2 diabetes. Diabetes Care. 2009;32:791-6.

11. Kahleova H, Matoulek M, Malinska H, Oliyarnik O, Kazdova L, Neskudla T, Skoch A, Hajek M, Hill M, Kahle M, Pelikanova T. Vegetarian diet improves insulin resistance and oxidative stress markers more than conventional diet in subjects with Type 2 diabetes. Diabet Med. 2011;28:549-59. 
12. Klementova M, Thieme L, Haluzik M, Pavlovicova R, Hill M, Pelikanova T, Kahleova HA. Plant-Based Meal Increases Gastrointestinal Hormones and Satiety More Than an Energy- and MacronutrientMatched Processed-Meat Meal in T2D, Obese, and Healthy Men: A Three-Group Randomized Crossover Study. Nutrients 2019; 11.

13. Kahleova H, Tura A, Hill M, Holubkov R, Barnard ND. A Plant-Based Dietary Intervention Improves Beta-Cell Function and Insulin Resistance in Overweight Adults: A 16-Week Randomized Clinical Trial. Nutrients 2018; 10.

14. Kahleova H, Tintera J, Thieme L, Veleba J, Klementova M, Kudlackova M, Malinska H, Oliyarnyk O, Markova I, Haluzik M, Pavlovicova R, Hill M, Tura A, Pelikanova T. A plant-based meal affects thalamus perfusion differently than an energy- and macronutrient-matched conventional meal in men with type 2 diabetes, overweight/obese, and healthy men: A three-group randomized crossover study. Clin Nutr. 2020 Oct 9:S0261-5614(20)30532-X. doi: 10.1016/j.clnu.2020.10.005.

15. Thornalley PJ, Langborg A, Minhas HS. Formation of glyoxal, methylglyoxal and 3-deoxyglucosone in the glycation of proteins by glucose. Biochem J. 1999;344(Pt 1):109-16.

16. Mullerova D, Tychtl Z, Muller L, Brazdova Z. NutriDan 1.2. Prague. Czech Republic: Danone Institute; 2003.

17. Meloun M, Hill M, Militky J, Kupka K. Transformation in the PC-aided biochemical data analysis. Clin Chem Lab Med. 2000;38:553-9.

18. Kahleova H, Tura A, Klementova M, Thieme L, Haluzik M, Pavlovicova R, Hill M, Pelikanova TA. PlantBased Meal Stimulates Incretin and Insulin Secretion More Than an Energy- and MacronutrientMatched Standard Meal in Type 2 Diabetes: A Randomized Crossover Study. Nutrients 2019; 11.

19. Li W, Lin L, Malhotra R, Yang L, Acharya R, Poss M. A computational framework to assess genomewide distribution of polymorphic human endogenous retrovirus-K In human populations. PLoS Comput Biol. 2019;15:e1006564.

20. Testa R, Bonfigli AR, Prattichizzo F, La Sala L, De Nigris V, Ceriello A. The "Metabolic Memory" Theory and the Early Treatment of Hyperglycemia in Prevention of Diabetic Complications. Nutrients 2017; 9.

21. Zhou Y, Jiang Z, Lu H, Xu Z, Tong R, Shi J, Jia G. Recent Advances of Natural Polyphenols Activators for Keap1-Nrf2 Signaling Pathway. Chem Biodivers. 2019;16:e1900400.

22. Yahfoufi N, Alsadi N, Jambi M, Matar C The Immunomodulatory and Anti-Inflammatory Role of Polyphenols. Nutrients 2018; 10.

23. Koch W. Dietary Polyphenols-Important Non-Nutrients in the Prevention of Chronic Noncommunicable Diseases. A Systematic Review. Nutrients 2019; 11.

24. Padh H, Subramoniam A, Aleo JJ. Glucose inhibits cellular ascorbic acid uptake by fibroblasts in vitro. Cell Biol Int Rep. 1985;9:531-8.

25. Margioris AN. Fatty acids and postprandial inflammation. Curr Opin Clin Nutr Metab Care. 2009;12:129-37. 
26. Nappo F, Esposito K, Cioffı M, Giugliano G, Molinari AM, Paolisso G, Marfella R, Giugliano D. Postprandial endothelial activation in healthy subjects and in type 2 diabetic patients: role of fat and carbohydrate meals. J Am Coll Cardiol. 2002;39:1145-50.

27. Esposito K, Nappo F, Marfella R, Giugliano G, Giugliano F, Ciotola M, Quagliaro L, Ceriello A, Giugliano D. Inflammatory cytokine concentrations are acutely increased by hyperglycemia in humans: role of oxidative stress. Circulation. 2002;106:2067-72.

28. Zhao S, Chen Z, Zheng J, Dai J, Ou W, Xu W, Ai Q, Zhang W, Niu J, Mai K, Zhang Y. Citric acid mitigates soybean meal induced inflammatory response and tight junction disruption by altering TLR signal transduction in the intestine of turbot, Scophthalmus maximus L. Fish. Shellfish Immunol. 2019;92:181-7.

29. Coronado M, Solis CJ, Hernandez PP, Feijoo CG. Soybean Meal-Induced Intestinal Inflammation in Zebrafish Is T Cell-Dependent and Has a Th17 Cytokine Profile. Front Immunol. 2019;10:610.

30. Sanchez A, Hubbard RW. Plasma amino acids and the insulin/glucagon ratio as an explanation for the dietary protein modulation of atherosclerosis. MedHypotheses. 1991;35:324-9.

31. Chen Z, Zuurmond MG, van der Schaft N, Nano J, Wijnhoven HAH, Ikram MA, Franco OH, Voortman T. Plant versus animal based diets and insulin resistance, prediabetes and type 2 diabetes: the Rotterdam Study. Eur J Epidemiol. 2018;33:883-93.

32. Paniagua JA. Nutrition, insulin resistance and dysfunctional adipose tissue determine the different components of metabolic syndrome. World J Diabetes. 2016;7:483-514.

33. Unger RH, Zhou YT. Lipotoxicity of beta-cells in obesity and in other causes of fatty acid spillover. Diabetes. 2001;50(Suppl 1):118-21.

\section{Tables}

TABLE 1: General characteristics of the study population.

Adapted and published in our previous part of the study [13].

Significant difference between the groups of participants: * is used for difference between T2 T2D and obese participants (* denotes $\mathrm{p} \otimes 0.05, * \star \star$ denotes $\mathrm{p} \otimes 0.001$ ), 9 is used for difference between obese and

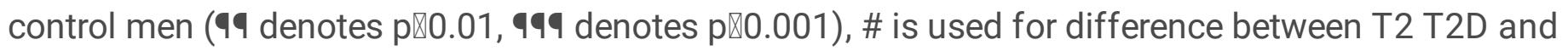
control men (\#\# denotes $\mathrm{p} \otimes 0.01$, \#\#\# denotes $\mathrm{p} \otimes 0.001$ ). 


\begin{tabular}{|c|c|c|c|}
\hline & $\begin{array}{l}\text { T2D } \\
n=20\end{array}$ & $\begin{array}{l}\text { Obese } \\
n=20\end{array}$ & $\begin{array}{l}\text { Controls } \\
n=20\end{array}$ \\
\hline Age (years) & $47.8 \pm 8.2$ & $43.0 \pm 7.0$ & $42.7 \pm 7.1^{\#}$ \\
\hline Weight (kg) & $108.2 \pm 11.9$ & $103.4 \pm 13.3^{\mathbf{4 9 9}}$ & $77.4 \pm 8.1^{\# \# \#}$ \\
\hline Body mass index $\left(\mathrm{kg} / \mathrm{m}^{2}\right)$ & $34.5 \pm 11.9$ & $32.7 \pm 3.9^{494}$ & $23.8 \pm 1.5^{\# \# \#}$ \\
\hline Waist circumference $(\mathrm{cm})$ & $106.9 \pm 23.6$ & $109.0 \pm 8.5^{\mathbf{4 q 9}}$ & $85.0 \pm 5.3^{\# \# \#}$ \\
\hline $\begin{array}{l}\mathrm{HbA} 1 \mathrm{c}(\mathrm{mmol} / \mathrm{mol}) \\
\text { Fasting plasma glucose }(\mathrm{mmol} / \mathrm{l})\end{array}$ & $\begin{array}{l}48.5 \pm 8.1^{\star \star \star} \\
7.2 \pm 1.5^{\star \star \star}\end{array}$ & $36.4 \pm 3.0$ & $36.1 \pm 3.2^{\# \# \#}$ \\
\hline Triglycerides (mmol/l) & $2.1 \pm 1.1$ & $5.1 \pm 0.3$ & $5.1 \pm 0.4^{\# \# \#}$ \\
\hline LDL-cholesterol (mmol/l) & $2.6 \pm 0.1^{*}$ & $2.2 \pm 1.1^{7}$ & $1.1 \pm 0.6^{\# \#}$ \\
\hline HDL-cholesterol (mmol/l) & $1.0 \pm 0.2$ & & $2.8 \pm 0.7$ \\
\hline Systolic blood pressure $(\mathrm{mm} \mathrm{Hg})$ & $144.4 \pm 13.4^{*}$ & $1.0 \pm 0.3^{\mathbf{4 9 9}}$ & $1.5 \pm 0.2^{\# \# \#}$ \\
\hline Diastolic blood pressure $(\mathrm{mm} \mathrm{Hg})$ & $96.2 \pm 8.8^{*}$ & $134.8 \pm 7.6^{49}$ & $124.0 \pm 11.4^{\# \# \#}$ \\
\hline Duration of diabetes (years) & $4.3 \pm 3.3$ & $\begin{array}{l}90.0 \pm 6.8^{\mathbf{4 9 9}} \\
-\end{array}$ & $80.7 \pm 5.6 \# \#$ \\
\hline
\end{tabular}

TABLE 2: Composition of the test meals.

The postprandial state was measured after intake of a standard breakfast - one of two energy- and macronutrient-matched meals in a random order: either a plant-based meal ( $V$-meal; tofu burger with spices, ketchup, mustard, tomato, lettuce and cucumber in a wheat bun) together with $300 \mathrm{~mL}$ of green tea, or a conventional meat meal (M-meal; cooked-pork seasoned meat in a wheat bun, tomato, cheddartype cheese, lettuce, spicy sauce) together with $300 \mathrm{~mL}$ Café Latte [14]. 


\begin{tabular}{|lll|}
\hline & V-meal & M-meal \\
\hline Total weight $(\mathrm{g})$ & 200 & 200 \\
Energy content (kJ/kcal) & $2154(514.9)$ & $2149(513.6)$ \\
Carbohydrates (g) (\%) & $54.2(44 \%)$ & $55(44.8 \%)$ \\
Proteins (g) (\%) & $19.9(16.2 \%)$ & $20.5(16.7 \%)$ \\
Total fat (g) (\%) & $22.8(39.8)$ & $22(38.6 \%)$ \\
Saturated fatty acids (g) & 2.2 & 8.6 \\
Fibre (g) & 7.8 & 2.2 \\
\hline
\end{tabular}

\section{Supplementary File}

Figure $\mathrm{S} 1$ is not available with this version.

\section{Figures}

\section{Image not available with this version}

Figure 1

Postprandial changes in plasma concentrations of glucose and lipids in T2D, obese and control subjects after a standard meal test consisting of either a conventional meat burger (M-meal) or a plant-based tofu burger ( $\mathrm{V}$-meal). Data are expressed as the mean with a $95 \% \mathrm{Cl}$. (A) Plasma glucose meal factor: $\mathrm{p}=0.309$ (T2D), $p=0.06$ (obese), $p=0.014$ (controls); group factor: $p \otimes 0.001$; (B) plasma triglycerides meal factor: $p=0.259$ (T2D), $p=0.004$ (obese), $p=0.432$ (controls); group factor: $p \otimes 0.001$; (C) NEFA meal factor: $p=0.432$

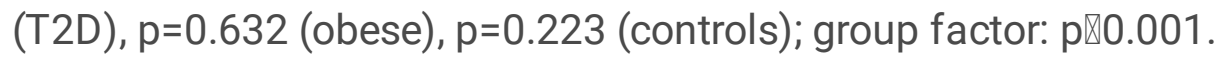

\section{Image not available with this version}

Figure 2 
Postprandial changes in plasma parameters of oxidative and dicarbonyl stress in T2D, obese and control subjects after a standard meal test consisting of either a conventional meat burger (M-meal) or a plantbased tofu burger (V-meal). Data are expressed as the mean with a $95 \% \mathrm{Cl}$. (A) Plasma GSH meal factor:

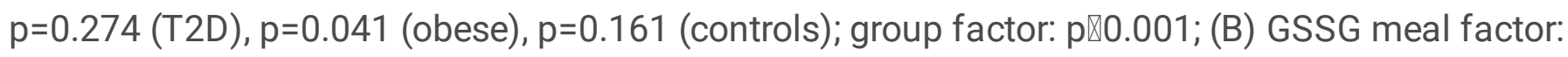
$p \varangle 0.001$ (T2D), $p=0.62$ (obese), $p=0.166$ (controls); group factor: $p \otimes 0.001$; (C) GPx activity meal factor: $p=0.045$ (T2D), $p=0.156$ (obese), $p=0.901$ (controls); group factor: $p \otimes 0.031$; (D) methylglyoxal meal factor: $p=0.058$ (T2D), $p=0.023$ (obese), $p=0.716$ (controls); group factor: $p \otimes 0.001 ;(E)$ ascorbic acid meal factor:

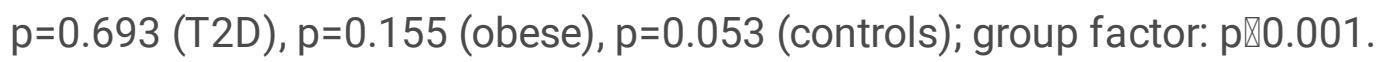

\section{Image not available with this version}

\section{Figure 3}

Postprandial changes in plasma inflammatory markers and appetite hormones in T2D, obese and control subjects after a standard meal test consisting of either a conventional meat burger (M-meal) or a plantbased tofu burger ( $\mathrm{V}$-meal). Data are expressed as the mean with a $95 \% \mathrm{Cl}$. (A) TNFa meal factors:

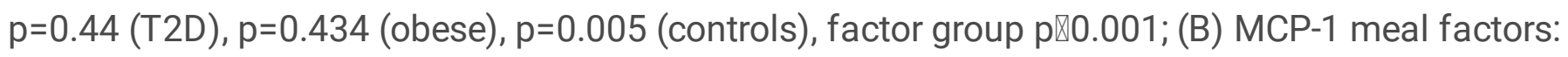
$p=0.671$ (T2D), $p=0.228$ (obese), $p=0.23$ (controls), factor group $p \otimes 0.001 ;(C)$ leptin meal factors: $p=0.002$

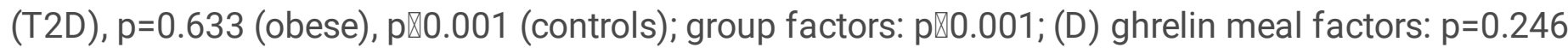
(T2D), $p=0.124$ (obese), $p=0.201$ (controls); group factors: $p \llbracket 0.001$. 